\title{
Efficacy of a Dermocosmetic Serum Combining Bakuchiol and Vanilla Tahitensis Extract to Prevent Skin Photoaging in vitro and to Improve Clinical Outcomes for Naturally Aged Skin
}

This article was published in the following Dove Press journal:

Clinical, Cosmetic and Investigational Dermatology

\author{
Daniel Bacqueville (1D ${ }^{1} *$ \\ Anne Maret ${ }^{2, *}$ \\ Maïté Noizet ${ }^{\prime}$ \\ Laure Duprat' \\ Christine Coutanceau (iD) ${ }^{3}$ \\ Victor Georgescu ${ }^{3}$ \\ Sandrine Bessou-Touya' \\ Hélène Duplan' \\ 'Department of Pharmacology, Pierre \\ Fabre Dermo-Cosmétique, Toulouse, \\ France; ${ }^{2}$ Clinical Skin Research Center, \\ Pierre Fabre Dermo-Cosmétique, \\ Toulouse, France; ${ }^{3}$ Laboratoires \\ Dermatologiques Avène, Lavaur, France
}

*These authors contributed equally to this work
Correspondence: Daniel Bacqueville Department of Pharmacology, Pierre Fabre Dermo-Cosmétique, Avenue

Hubert Curien, BP I3562, 31035

Toulouse Cedex I, France

Tel +33534506427

Fax +33 534503424

Email daniel.bacqueville@pierre-fabre.com
Background: Skin aging is characterized by slacking and loss of density, especially under ultraviolet (UV) radiation exposure.

Objective: To investigate the beneficial effects of a combination containing bakuchiol (BK) and vanilla tahitensis extract (VTE) to prevent skin photoaging in vitro and to improve clinical outcomes for naturally aged skin.

Materials and Methods: Human dermal fibroblasts were treated with active compounds, exposed to an acute dose of UVA and analyzed by confocal microscopy: actin network for morphology, interleukin-8 (IL-8) for inflammation and p16 for senescence. Human skin was used to evaluate chronic UVA-induced glycosaminoglycan (GAG) loss and to assess the benefit of topical application of a BK+VTE serum (Alcian blue staining). An open-label clinical trial was conducted in women applying the serum twice daily for 56 days $(n=43)$. Skin remodeling was assessed by FaceScan ${ }^{\circledR}$. Firmness was evaluated through Dynaskin ${ }^{\circledR}$ and clinical scoring. Skin radiance was also rated on standardized full-face photographs.

Results: UVA induced a significant increase in IL-8 and p16 expression and marked morphological changes in fibroblasts. Treatment with BK or VTE alone prevented both actin network alteration and IL-8 upregulation. Interestingly, BK+VTE demonstrated synergistic protection against IL-8 and p16 overexpression. Serum application prevented GAG loss at the dermo-epidermal junction and increased dermal GAG in UVA-exposed skin explants. In the clinical trial, face ptosis was reduced by $11 \%$ on average for 26 responsive subjects and up to $23 \%$. Depth of skin deformation was also reduced by $24 \%$ on average for 30 responsive subjects and up to $30 \%$. This firming effect was confirmed by clinical scoring. Radiance was significantly improved by $29 \%$ on average for 33 responsive subjects. The serum demonstrated good tolerance/safety.

Conclusion: BK+VTE combination demonstrated anti-aging efficacy and might provide a substantial benefit in the daily care of naturally aged skin in women, through their synergistic effect on inflammaging and senescence.

Keywords: anti-aging therapy, clinical trial, inflammaging, senescence, skin model

\section{Introduction}

Skin aging is a complex, multifactorial process which is influenced by genetic, environmental, mechanical and socioeconomic factors. ${ }^{1,2}$ Intrinsic or chronological structural changes of skin aging include increased skin fragility, decreased ability of the skin to heal, and increased risk of toxicological injury. Photoaging involves the 
premature aging of skin due to cumulative exposure to ultraviolet (UV) radiation and the amount of melanin in the skin. ${ }^{3,4}$ These changes are associated with the development of a range of cutaneous disorders, varying from benign to malignant, and they produce undesirable clinical signs of aging such as wrinkling, uneven pigmentation, and loss of density and volume. ${ }^{5,6}$

Skin aging results from the disruption of fibroblastextracellular matrix interactions. ${ }^{7}$ It is largely characterized by atrophy due to a loss of/damage to collagen, increased production of matrix metalloproteinases, decreased fibroblast cell density and morphology, degeneration of the elastic fiber network and reduced skin hydration. ${ }^{4,7,8}$ Aging is also closely associated with alterations in the immune system and inflammation (inflammaging). For example, the aged dermis presents more mast cells and neutrophils. ${ }^{6}$ This phenomenon is correlated with an upregulation of interleukin-8 (IL-8), a proinflammatory and chemotactic chemokine, in aged skin. ${ }^{9}$ Although monocytes are the main source of IL-8, it is also produced by macrophages, endothelial cells, keratinocytes and fibroblasts. Due to their location, production of IL-8 by fibroblasts could play an important role in communicating with dermal endothelial cells, increasing the migration of neutrophils into the dermis and boosting inflammation. Indeed, the inflammatory response of human skin fibroblasts to cellular stress is associated with increased IL- 8 production. ${ }^{10}$ Moreover, the synthesis of IL- 8 by fibroblasts is influenced considerably by UV irradiation. ${ }^{11}$

It is also acknowledged that aging is associated with the accumulation of senescent cells which present a specific phenotype: the secretion of cytokines and other mediators that influence skin functions (secretome) and the inability to divide in the tissue (cell cycle blockade). ${ }^{12}$ Thus, the cell cycle is a complex mechanism which is highly regulated by aging. Among the molecular markers of senescence, the p16 protein is a specific inhibitor of the cell cycle which stops cellular proliferation in the G1 phase. ${ }^{13,14}$ Consequently, p16 participates in the induction of senescence and, therefore, plays a role in cell aging. ${ }^{12}$

Aged skin also constitutively expresses reduced levels of glycosaminoglycans (GAGs), polysaccharide chains which comprise long repeats of specific disaccharide units. Proteoglycans (PGs), GAGs attached to a core protein, are distributed widely throughout the skin. ${ }^{15}$ GAGs are involved in structural and physiological regulatory roles in the skin. Because GAGs bind up to 1000 times their volume in water, skin hydration and density is associated with the content and distribution of dermal GAGs. ${ }^{16-18}$
Although there are many products which claim to have anti-aging properties, there is a need for innovative products which have been evaluated from a scientific and clinical basis. Bakuchiol (BK), a plant-based retinol-like alternative to retinol, has been shown to regulate the expression of genes and proteins involved in the skinaging process, and it significantly improves lines and wrinkles, pigmentation, elasticity, firmness and overall reduction in photo-damage without the undesirable effects usually associated with retinol therapy. ${ }^{19,20}$ Vanilla tahitensis extract (VTE) also possesses numerous medicinal properties such as antioxidant power and they are rich in polyphenols that are well known to improve the signs of skin aging. ${ }^{21}$

Therefore, the aim of the current study was to evaluate the anti-aging effects of a new combination of two active ingredients, BK and VTE, in in vitro and ex vivo studies and in a clinical study in women with naturally aged skin.

\section{Materials and Methods}

\section{Combination of Active Ingredients and}

\section{Serum Formulation}

Bakuchiol (BK; Sytenol ${ }^{\circledR}$, Sytheon Ltd) was purchased from Rahn France and was obtained from Psoralea coryfolia with a purity of about $95 \% .{ }^{19} \mathrm{BK}$ was used in cell culture at $0.5 \mu \mathrm{g} / \mathrm{mL}$ from a $10 \mathrm{mg} / \mathrm{mL}$ stock solution in dimethylsulfoxide. Vanilla tahitensis extract (VTE; Vanirea ${ }^{\circledR}$ ) was obtained from Solabia Ltd France. It is a vegetal active ingredient obtained via hydroglycolic extraction of mature tahitian vanilla pods (vanilla tahitensis fruit extract) using water and propylene glycol. VTE contains a high concentration of polyphenols including vanillin and parahydroxybenzoic acid and presents antioxidative activities. It was diluted at $0.05 \%$ in cell culture medium.

Both active ingredients were prepared at the same concentrations in combination studies. A dermocosmetic serum was also formulated with a combination of $1.5 \%$ $\mathrm{BK}$ and $1 \%$ VTE according to International Nomenclature of Cosmetic Ingredients nomenclature: Avene thermal spring water, caprylic/capric triglyceride, glycerin, methyl gluceth 20, coco caprylate/caprate, dimethicone, bakuchiol, glyceryl linoleate jojoba seed oil, 1,2-hexanediol caprylyl glycol cetyl alcohol fragrance, glyceryl linolenate, glyceryl oleate, glyceryl palmitate, glyceryl stearate, glycine soja oil, sunflower seed oil, hydrogenated polyisobutene, hydrogenated starch hydrolysate, hydroxyethyl acrylate/sodium acryloyldimethyl taurate, copolymer lactic 
acid, mica, palmitic acid, PEG-7, trimethylpropane, coconut ether propylene glycol, sodium stearoyl, glutamate sorbitan isostearate, stearic acid, titanium dioxide, tocopherol tocopheryl glucoside, tropolone and vanilla tahitensis fruit extract water.

\section{In vitro Evaluation of BK, VTE and Their Combination Using Irradiated Human Dermal Fibroblasts in a Skin Photoaging Model}

Normal human dermal fibroblasts (NHDFs) were obtained from three different donors (females 20-37 years old). Prior to sampling, each donor provided written donor permission according to French regulations on donor rights.

Cell samples were cultured at $37^{\circ} \mathrm{C}$ and $5 \% \mathrm{CO}_{2}$ in DMEM enriched with l-glutamine $2 \mathrm{mM}$, penicillin $50 \mathrm{U} / \mathrm{mL}$, streptomycin $50 \mathrm{mg} / \mathrm{mL}$ and fetal calf serum $1 \% .{ }^{20}$ NHDFs were seeded on 6-well plates and cultured in the media for $36 \mathrm{~h}$. Samples were treated with test compounds (BK, VTE or combined BK+VTE) for $1 \mathrm{~h}$ then irradiated in phosphate-buffered saline (PBS) using a Waldmann UVA lamp to deliver an acute UVA dose $\left(60 \mathrm{~J} / \mathrm{cm}^{2}\right)$. NHDFs were not incubated with active ingredients following UVA exposure and they were harvested $24 \mathrm{~h}$ after UVA irradiation for performing immunofluorescence analysis. ${ }^{23}$

Controls consisted of non-irradiated, non-treated NHDFs (no stress/no treatment), and UVA-irradiated, non-treated NHDFs (stress, no treatment).

For immunofluorescence analysis, NHDFs were labelled for actin (morphology), IL-8 (inflammation), and p16 (senescence), and nuclei were labelled with DAPI. Protocols used specific antibodies as described previously: ${ }^{23}$ actin green (Molecular Probes R37110, excitation 488nm/emission 500-550nm), anti-IL8 1/1500 (Abcam Ab18672), antip16INK4a 1/150 (Abcam Ab192053), alexa fluor 594coupled secondary antibody 1/1000 (Life Technologies A11020, excitation 561nm/emission $570-620 \mathrm{~nm}$ ) and NucBlue (Molecular Probes R37606, excitation 405nm/emission $425-475 \mathrm{~nm})$. Images were acquired using a confocal microscope (Nikon $\mathrm{A} 1 \mathrm{R}+$ ) and signals were quantified using NIS Element software.

Intergroup comparisons were performed using one-way ANOVA (repeated measures), completed by a Dunnett's post-test. Results were considered statistically significant if $P<0.05$ or $P<0.001$. Statistical analysis was performed using PRISM software.

\section{Evaluation of the Combination Using an ex vivo Human Skin Aging Assay}

To study the redensifying effect of a BK+VTE serum formulation, human skin explants $\left(1.12 \mathrm{~cm}^{2}\right)$ were obtained from three different donors, isolated during abdominoplasty surgery (females 32-53 years old). Each donor provided prior written donor permission according to French regulations on donor rights. Tissue culture was performed at $37^{\circ} \mathrm{C}$ and $5 \% \mathrm{CO}_{2}$ in complete DMEM $(1.5 \mathrm{~mL}) \cdot{ }^{22,23}$ In vitro photoaging stress was induced in the human explants using chronic UVA radiation. Human explants were transferred to PBS and irradiated $\left(12 \mathrm{~J} / \mathrm{cm}^{2}\right)$, at 4,24 and $30 \mathrm{~h}$ after culture initiation, using a Biosun Vilber Lourmat (Vilber, Eberhardzell, Germany) simulator at $365 \mathrm{~nm}$ wavelength, according to standard procedures. ${ }^{22}$ Serum $\left(5 \mathrm{mg} / \mathrm{cm}^{2}\right)$ was applied topically after each UVA exposure and $24 \mathrm{~h}$ after the last exposure. Skin explants were harvested $48 \mathrm{~h}$ after the last UVA exposure, fixed, and embedded in paraffin. As a marker of the extracellular matrix for evaluating dermal density, GAG content was evaluated with Alcian blue staining.

Controls consisted of non-irradiated, non-treated explants (no stress/no treatment), and UVA-irradiated, nontreated explants (stress, no treatment).

\section{Clinical Evaluation of a Serum Formulation of the Combination in Healthy Volunteers}

The anti-aging effects of both active ingredients, combined in a serum formulation, were evaluated in an open-label, singlecenter clinical trial in 43 women with naturally aged skin. They applied the serum twice daily (morning, evening) in the normal conditions of use, for 56 days on the whole face, neck and neckline. Subjects could apply their usual moisturizing cream (without an anti-aging claim) 15 min after application of the investigational product. A minimum of $20 \%$ of each skin type (normal, greasy, dry, combination) was represented in the study.

Subjects maintained a daily log recording: the number of daily investigational product applications; the nature (e.g. skin tension, stinging, etc.), intensity (very mild to severe), duration, and timing of occurrence of any discomfort and/or intolerance reactions; and details of any other medications taken.

Forty-three (43) healthy Caucasian females aged 45 to 65 years with skin phototypes II or III who had provided written informed consent were included in the study. 
Participants also provided consent for the use of their images in publication. Subjects had a lack of skin comfort, self-declared sensitive facial skin and presented a loss of facial skin density, skin sagging (minimum score of 2, Bazin scale ${ }^{24}$ ), a lack of skin radiance and a dull complexion. Subjects were required to have healthy skin on the face (free of eczema, wounds, inflammatory scars); had established and well-tolerated facial care, hygiene and make up habits, for $\geq 15$ days prior to study initiation; if non-menopausal, were using a method of contraception; if applicable, were receiving stable systemic treatment for $\geq 1$ month before study initiation; and were committed to maintaining their life habits (e.g. sport, diet, smoking and sleep pattern) during the study. Participants were not allowed to use cosmetic or oral anti-aging products, other than the dermocosmetic serum being tested, during the study. No topical or oral retinoids could be used within 4 or 6 months, respectively, of commencing study participation.

Efficacy assessments were performed at the investigational center on baseline (before first application) and after 28 and 56 days, of twice daily use under normal conditions. Prior to assessment, no other product was applied to the face, neck and neckline since the previous evening.

The remodeling effect was evaluated using the fringe projection system FaceScan ${ }^{\circledR}$ (GFM, Germany), ${ }^{25}$ on the whole face. This standard method consists in a fringe pattern projection system recorded by a digital camera enabling creation of high-resolution 2D images of a subject's face. It also allows a 3-dimensional (3D) data acquisition with a focus on the lower face to assess the jowl ptosis.

Skin firmness was measured by two validated methods. The first one was Dynaskin ${ }^{\circledR}$ (EoTech, France), ${ }^{26}$ in which an air stream perpendicular to the skin surface was blown into the hollow of the cheek, without skin contact, causing skin deformation, that is inversely proportional to skin firmness. The shape of the local skin surface was captured before, during and after deformation with a 3D sensor using the fringe projection technique. The second one was clinical scoring by a board-certified dermatologist using an 11-point scale from 0 (no firmness) to 10 (very firm). Moreover, skin radiance was rated by five trained assessors on an 11-point scale from 0 (dull complexion) to 10 (radiant) using digital photographs of subject's face.

Statistical analyses of efficacy parameters were performed versus baseline using the Student's $t$-test or Wilcoxon test.
The study was conducted according to the Declaration of Helsinki (1964) and its successive updates, with adherence in the spirit of Guidance on Good Clinical Practice CPMP/ICH/135/95(R2). This study, performed on a cosmetic product encompassed by article L. 5131-1 of the French Public Health Code, is in accordance with Decree number 2017-884 (9 May 2017) modifying some regulatory requirements concerning research involving human subjects. This study follows the article of French law number 2012-300 (5 March 2012) relative to research involving human subjects. Consequently, the study did not require ethics committee approval and competent authority authorization.

\section{Results}

\section{In vitro Evaluation of BK, VTE and Their Combination Using Irradiated Human Dermal Fibroblasts in a Skin Photoaging Model}

Using an in vitro human dermal fibroblast model, the benefits of both active ingredients were demonstrated for three major markers associated with skin aging: morphology (actin), inflammation (IL-8) and senescence (p16).

\section{Cellular Morphology}

Actin staining showed that UVA irradiation induced marked morphological changes in NHDFs, from a starshaped pattern to a fusiform pattern, mimicking fibroblast alteration during photoaging with a loss of extracellular matrix production and the classical structure (Figure 1, green labelling). Treatment with the individual compounds, and their combination, prevented the morphological changes induced by acute UVA exposure, resulting in improved fibroblast morphology. The cellular structure was comparable to that of observed in control, nonirradiated NHDFs (Figure 1, green labelling).

\section{Cellular Inflammation}

UVA irradiation of NHDFs induced a significant increase in IL-8 expression compared with non-irradiated cells, mimicking the induction of inflammation experienced during photoaging with increased cellular IL8 production (inflammaging) (Figure 1, red labelling). Treatment with VTE $0.05 \%$ or BK $0.5 \mu \mathrm{g} / \mathrm{mL}$ markedly reduced IL-8 expression induced by acute UVA exposure (Figure 1, red labelling). As shown in Figure 2A, protection was $83.8 \%$ and $88.3 \%$, respectively $(P<0.05)$. However, 


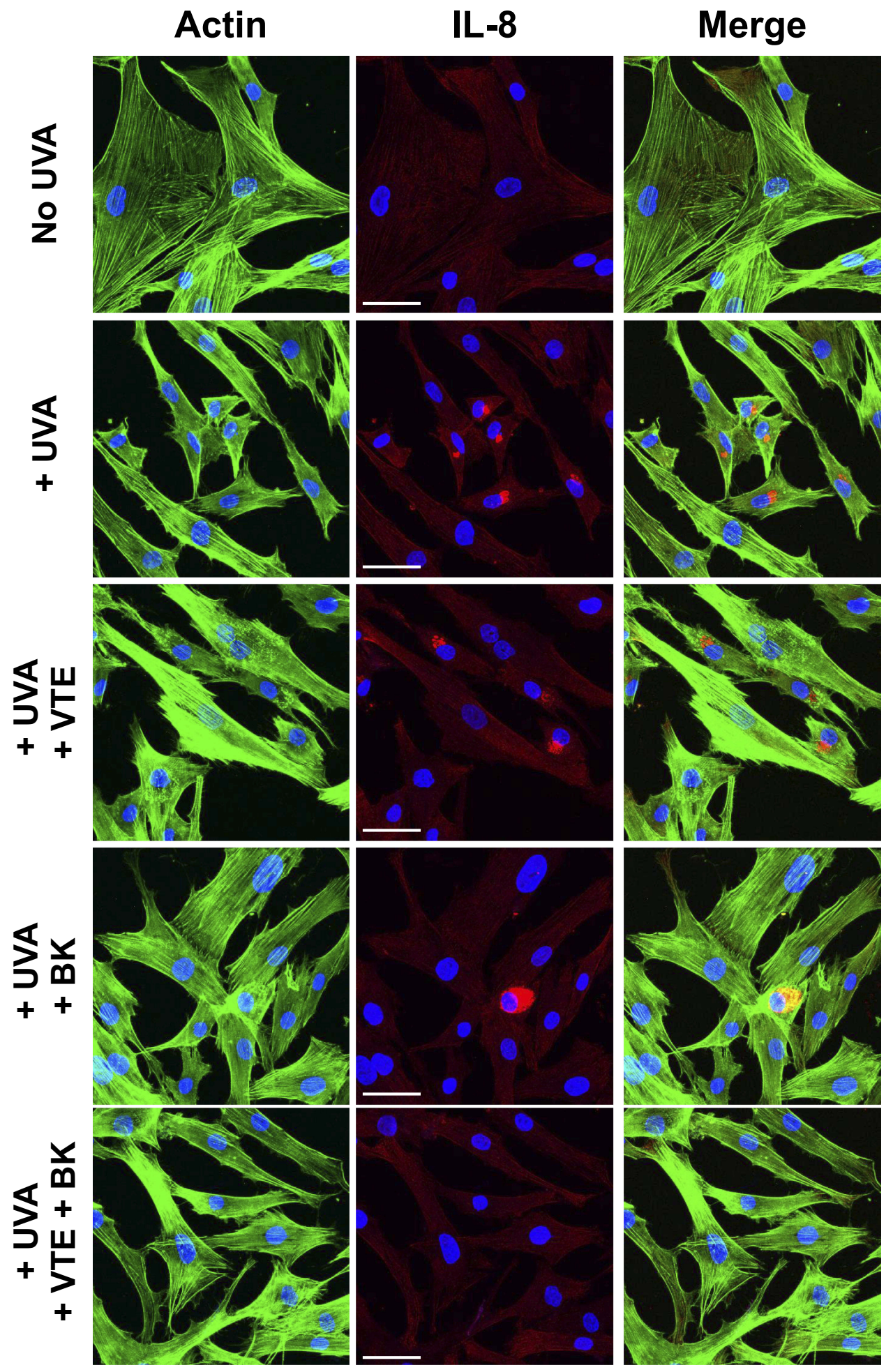

Figure I Combination of BK+VTE improves UVA-induced morphology alteration and inflammation in human dermal fibroblasts. Fibroblasts were treated in the presence or absence of VTE or BK alone or in combination. Cells were then irradiated using UVA and analysed by laser scanning confocal microscopy. Pictures show immunofluorescence staining of actin network (green labelling) and interleukin-8 (red labelling) as markers of morphology and inflammation, respectively. Cultures were counter-stained with DAPI to locate the nuclei of fibroblasts (blue labelling). Scale bar $=50 \mu \mathrm{m}$.

combination of both active ingredients resulted in a significant synergistic reduction of IL-8 expression induced by UVA exposure, to levels which were identical to that of observed in control, non-irradiated cells (Figure 1, red labelling). Protection reached 95.1\% $(P<0.05)$ with the BK+VTE combination (Figure $2 \mathrm{~A})$. 

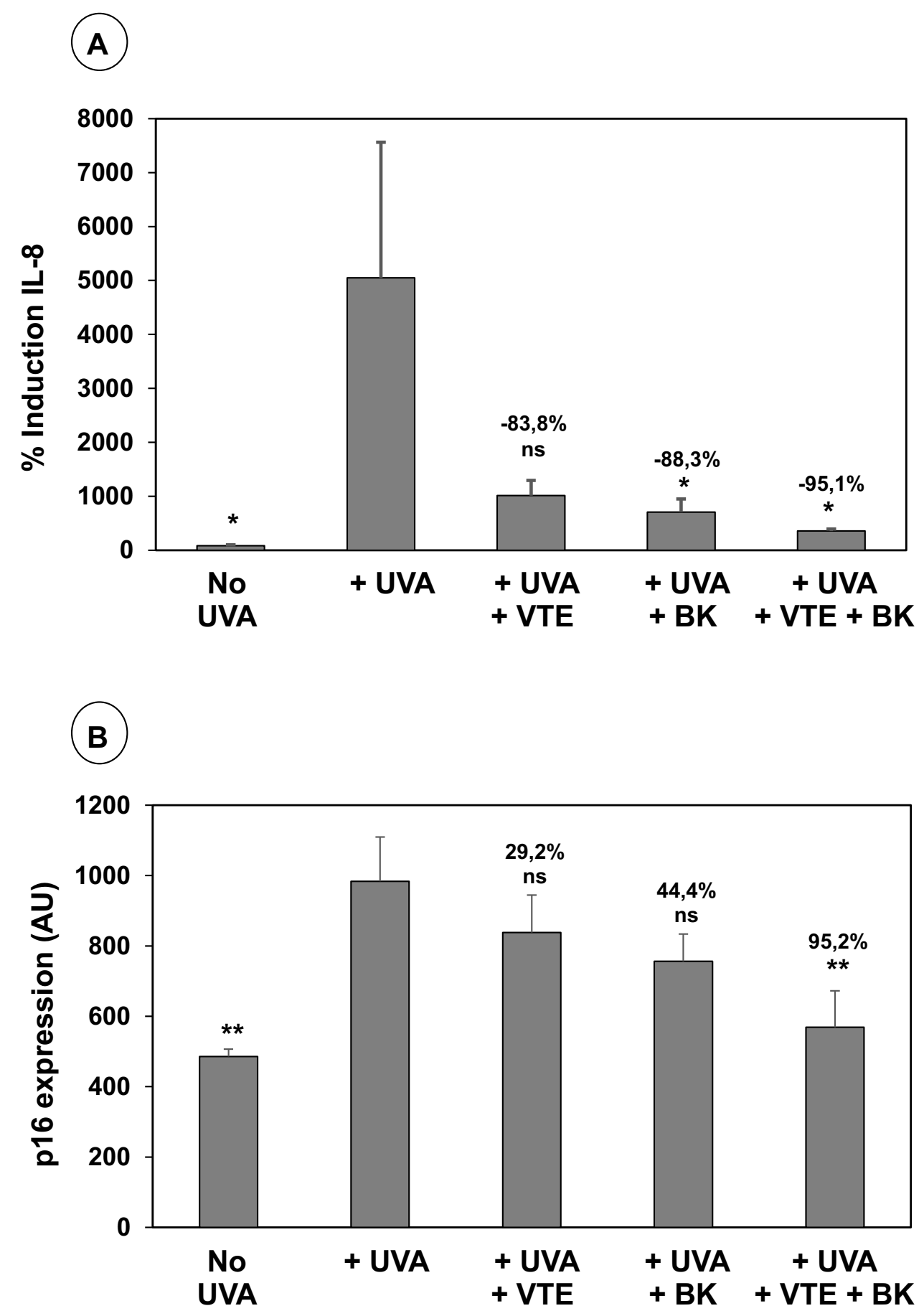

Figure 2 Quantitative analyses of the effects of BK+VTE combination on IL-8 and pl 6 immunolabelling. (A) IL-8 quantification from representative pictures obtained from 3 independent donors. Results are expressed in \% induction IL-8 mean \pm SEM. Statistics were performed using one-way ANOVA completed by a Dunnett's vs non-treated UVA. ns $p>0.05 ;{ }^{*} p<0.05$. \% indicates active ingredient protection. (B) pl6 quantification from representative pictures obtained from 3 independent donors. Results are expressed in pl 6 expression arbitrary unit (AU) mean \pm SEM. Statistics were performed using a one-way ANOVA completed by a Dunnett's vs non-treated UVA. ns $P>0.05$; ** $\mathrm{p}<0.00 \mathrm{I}$. \% Indicates active ingredient protection.

\section{Cellular Senescence}

UVA irradiation of NHDFs induced increased p16 expression, mimicking the induction of senescence experienced during photoaging with increased p16 expression in cell nuclei (Figure 3, red labelling). Treatment with VTE $0.05 \%$ or BK
$0.5 \mu \mathrm{g} / \mathrm{mL}$ resulted in a tendency to prevent p16 expression (Figure 3, red labelling). As indicated in Figure 2B, protection was $29.2 \%$ and $44.4 \%$, respectively $(P>0.05)$. However, combination of both active ingredients improved fibroblast senescence and significantly decreased p16 levels to those found in 

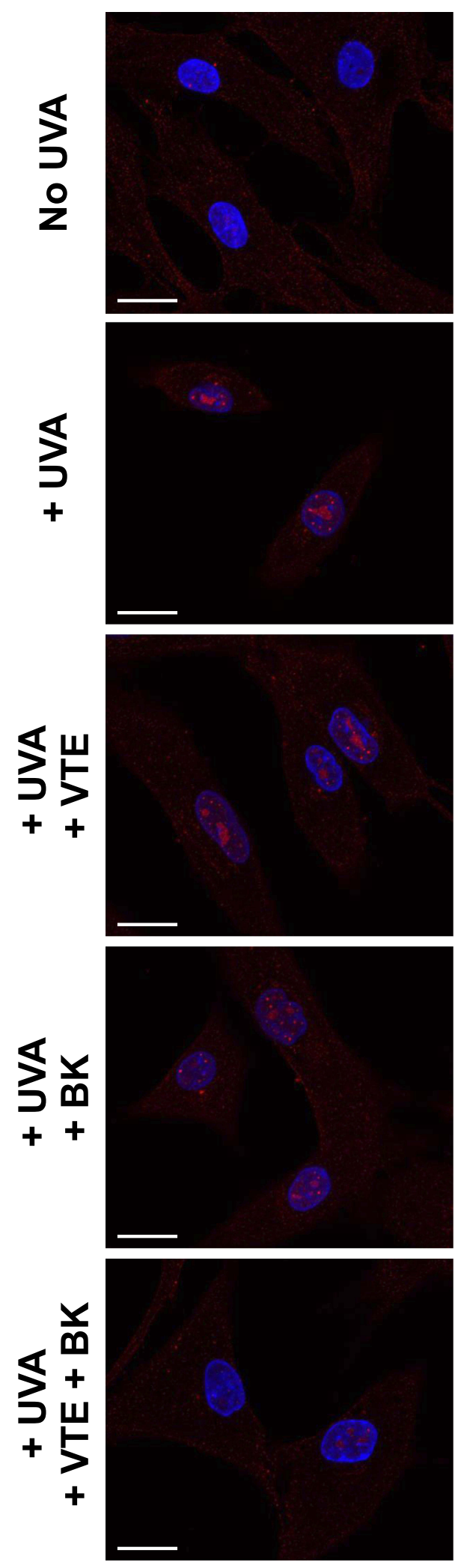

Figure 3 Combination of BK+VTE improves UVA-induced senescence in human dermal fibroblasts. Fibroblasts were treated in the presence or absence of VTE or BK alone or in combination. Cells were then irradiated using UVA and analysed by laser scanning confocal microscopy. Pictures show immunofluorescence staining of the pl6 protein as a nuclear marker of senescence (red labelling). Cultures were counterstained with DAPI to locate the nuclei of fibroblasts (blue labelling). Scale bar $=20 \mu \mathrm{m}$. control, non-irradiated cells (Figure 3, red labelling). Protection reached a synergistic effect of $95.2 \%(P<0.001)$ with the BK+VTE combination, higher than the addition of the individual effects (Figure 2B).

\section{Effect of the Combined BK+VTE Formulated as a Serum on Dermal Density Using an ex vivo Human Skin Aging Assay}

A photodamaged human skin model was used to mimic dermal alterations induced by a chronic UVA exposure during photoaging with a loss of extracellular matrix production, especially GAGs. They were specifically stained in blue color by Alcian blue (Figure 4). In non-irradiated skin control, the GAGs were mainly located at the dermoepidermal junction (DEJ) as a thin intense blue line just beneath the epidermis (seen in pink color). Low magnification showed a uniform staining along with the epidermis (Figure 4 left panel). The underlying papillary dermis was also positive for the presence of GAGs. Staining was better visualized at higher magnification (Figure 4 right panel). In contrast, chronic UVA impaired GAG content and induced a strong disruption of GAG network at the DEJ with a more diffuse staining and a much less intense blue color (Figure 4 arrows). The loss of GAGs was especially observed at high magnification and was associated with an alteration of the collagen/elastic fibers in the papillary dermis. Treatment with the BK+VTE dermocosmetic serum improved dermal density associated with a recovery of GAG content. Indeed, the organization of the GAG network of both irradiated and BK+VTE-treated skin samples was almost identical to that of nonirradiated control samples (Figure 4 left panel). The blue color intensity was also comparable between the control skin and the serum-treated skin exposed to UVA (Figure 4 right panel). Thus, the BK+VTE serum protected the skin from UVA-induced GAG alterations and prevented the loss of dermal density.

\section{Clinical Evaluation of the Combined BK + VTE Serum Anti-Aging Effect in Healthy Volunteers}

The effects of the serum were evaluated on skin remodeling, firmness and radiance in healthy women $(n=43$ [Day 1]; mean age 58 [range 45-65] years). Skin phototype was II (43\%) or III (57\%) and skin type was classified 



Figure 4 Combined BK+VTE, formulated as a serum, improves dermal density in an ex vivo human skin aging assay. Skin explants were chronically exposed to UVA radiation. After each UVA dose, BK+VTE serum was applied topically onto the skin surface. Tissues were then processed by routine Alcian blue staining to evaluate the GAG content as a marker of the extracellular matrix. Pictures were acquired by photon microscopy. Arrows indicate GAG loss at the dermo-epidermal junction. Left panels show low magnification with scale bar at $100 \mu \mathrm{m}$. Right panels show high magnification with scale bar at $50 \mu \mathrm{m}$.

as normal $(23.8 \%)$, greasy $(26.2 \%)$, dry $(26.2 \%)$ or combination (23.8\%). Day 28 and Day 56 assessments were performed for 42 and 41 women, respectively. The results were compared to baseline parameters to evaluate the invivo efficacy of the combination BK+VTE.

The assessment of the anti-sagging effect of the serum using FaceScan ${ }^{\circledR}$ showed a significant decrease in mean ptosis volumes of the lower part of face after 56 days of application compared to baseline $(P<0.0001$; Table 1$)$. This indicates a more defined face contour line, especially on jowl part. Overall, $63 \%$ of subjects had a significant remodeling effect after 56 days of daily use under normal conditions. The data of a representative subject are shown in Table 1 and Figure 5.

The assessment of skin firmness with DynaSKIN ${ }^{\circledR}$ showed that the daily use of serum led to a significant reduction of both depth and volume of skin deformation from baseline to Day 56 ( $P<0.0001$ for each; Table 1$)$. The mean decrease in these parameters was $17 \%$ and $16 \%$, respectively, after 56 days, which demonstrated a significant firming effect of the serum. In total, $73 \%$ of subjects showed improvement in the depth of skin 
Table I Efficacy Outcomes for BK+VTE Serum in Healthy Female Volunteers

\begin{tabular}{|c|c|c|c|c|c|}
\hline Parameter & $\begin{array}{l}\text { Day I } \\
(n=43)\end{array}$ & $\begin{array}{l}\text { Day } 28 \\
(n=42)\end{array}$ & $\begin{array}{l}\text { Day } 56 \\
(n=4 I)\end{array}$ & $\Delta \mathrm{D} 28$ & $\Delta$ D56 \\
\hline \multicolumn{6}{|l|}{ Skin Remodeling } \\
\hline \multicolumn{6}{|l|}{ Ptosis Volume $(\mathrm{mL})-$ Facescan $^{\circledR}$} \\
\hline Mean & 1.84 & 1.81 & $\mathrm{I} .72 *$ & -0.04 & -0.12 \\
\hline Data subject described in Figure 5 & 3.37 & 2.95 & 2.6 & -0.42 & -0.77 \\
\hline \multicolumn{6}{|l|}{ Skin Firmness } \\
\hline \multicolumn{6}{|l|}{ Volume of the Skin Deformation $\left(\mathrm{mm}^{3}\right)-$ Dynaskin $^{\circledR}$} \\
\hline Mean & 100.2 & 96.4 & $84.0 *$ & -3.6 & -15.7 \\
\hline Data subject described in Figure 6 & 107.2 & 85.7 & 65.7 & -21.5 & -41.6 \\
\hline \multicolumn{6}{|l|}{ Maximum Depth of the Skin Deformation $(\mathrm{mm})-$ Dynaskin $^{\circledR}$} \\
\hline Mean & 0.74 & 0.7 & $0.62 *$ & -0.05 & -0.12 \\
\hline Data subject described in Figure 6 & 0.77 & 0.61 & 0.56 & -0.16 & -0.21 \\
\hline \multicolumn{6}{|l|}{ Clinical Scoring - Scale 0-10 } \\
\hline Mean & 4.1 & $4.9 *$ & $5.6^{*}$ & & \\
\hline \multicolumn{6}{|l|}{ Skin Radiance } \\
\hline \multicolumn{6}{|l|}{ Clinical Scoring - Scale 0-10 } \\
\hline Mean & 5.1 & $6.0^{*}$ & $6.1^{*}$ & & \\
\hline
\end{tabular}

Note: $* P<0.0001$ change from baseline (Day I).

deformation at Day 56 while $78 \%$ of them had significant decrease of skin volume deformation. The data of a representative subject are shown in Table 1 and Figure 6. This was confirmed by the results of clinical scoring of skin firmness by the dermatologist. Compared to baseline, the scores increased significantly by $17 \%$ and
$35 \%$ after 28 and 56 days, respectively $(P<0.0001)$ (Table 1). Overall, $95 \%$ of subjects showed a significant improvement in skin firmness as assessed by the dermatologist on Day 56.

Additionally, the serum application improved significantly skin radiance scores by $17 \%$ and $20 \%$ after 28 and
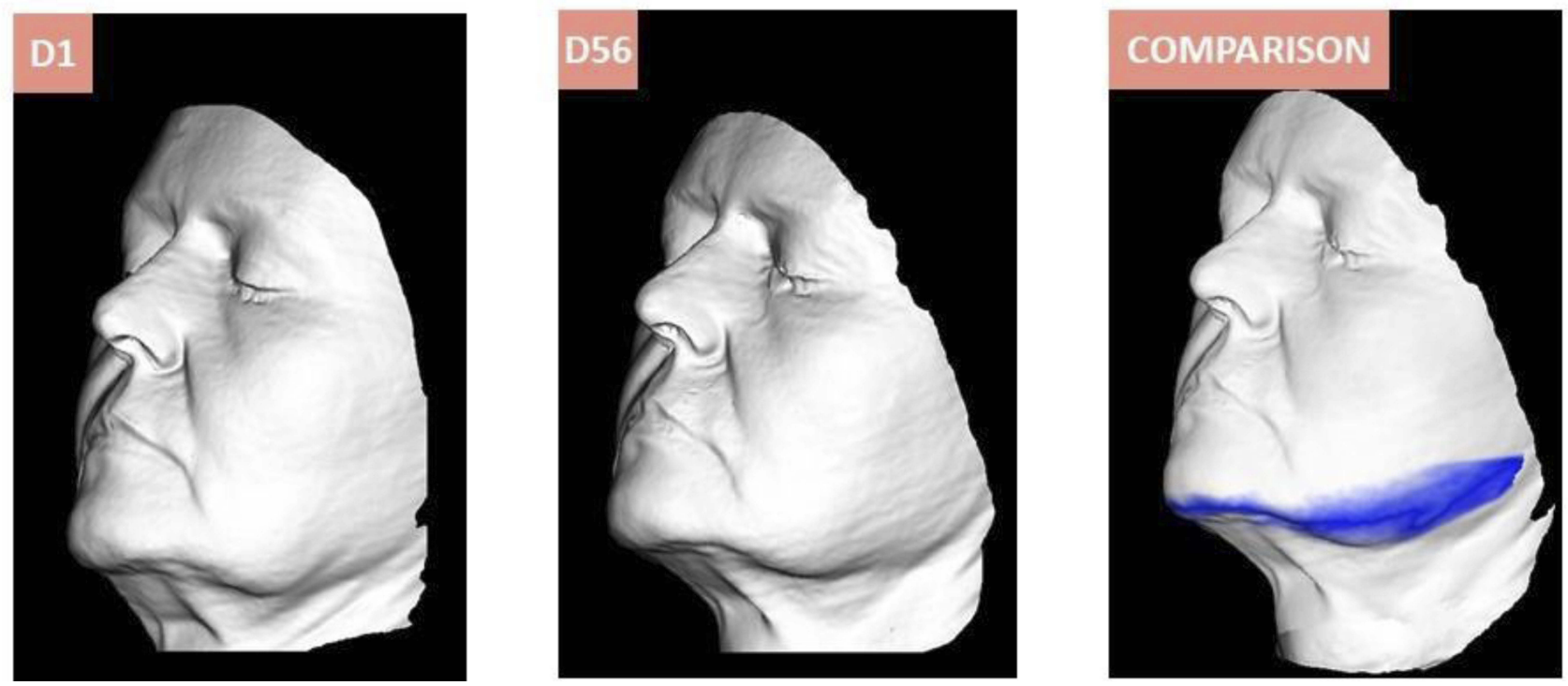

Figure 5 Illustrative analysis of improvement of ptosis volume by Facescan ${ }^{\circledR}$ following application of the BK+VTE serum. Blue area refers to decrease in ptosis volume and a more defined face contour, showing a remodeling effect of the product. Ptosis volume decreased by $22.8 \%$ from Day I to Day 56 . 

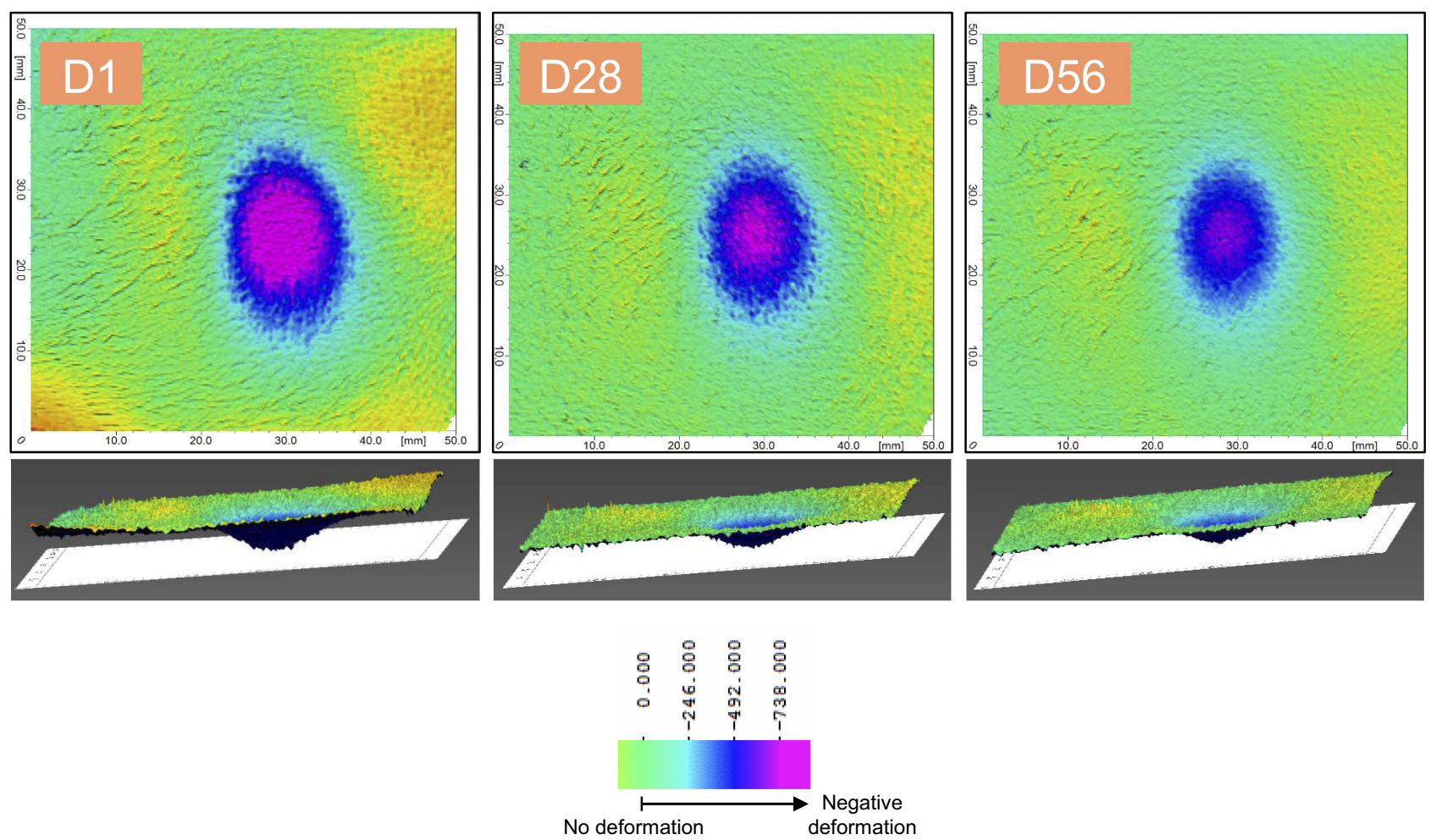

Figure 6 Illustrative results of skin firmness improvement by Dynaskin ${ }^{\circledR}$ following application of the BK+VTE serum. Cross-section showing the depth of skin deformation from pink color (deep deformation) to green color (no deformation) in the lower part of the cheek. Skin deformation is reduced after 28 and 56 days of treatment as demonstrated by the disappearance of the pink color which shows that skin is firmer. The image below shows another manner to confirm the reduction of skin deformation. From Day I to Day 56 , skin deformation depth was reduced by $30.4 \%$, and skin deformation volume by $36.7 \%$.

56 days, respectively $(P<0.0001$; Table 1$)$. Overall, the increase of skin radiance was significant in $80 \%$ of users on Day 56. The data of a representative subject are shown in Table 1 and Figure 7. Finally, global tolerance/safety based on the results of all studies performed with the dermocosmetic serum was judged as "very good" for skin evaluation and "excellent" for ophthalmologic evaluation (data not shown).

\section{Discussion}

The proportion of aging individuals is increasing worldwide. ${ }^{27}$ With increased aging populations, the medical importance of dermatological concerns continues to expand. The clinical signs of aging are important factors of well-being and, coupled with the increased aging population, there is a need for new, innovative anti-aging products. ${ }^{28}$ The current study demonstrated the anti-aging properties of an innovative combination of two active ingredients, BK and VTE, in experimental models and in female volunteers with naturally aged skin.

The BK+VTE combination demonstrated efficacy both in in vitro and ex vivo skin model, providing evidence for the in vivo mechanism of action of the dermocosmetic serum. In addition to preventing matrix degradation and morphological changes (actin), the BK +VTE combination demonstrated innovative synergistic benefits on inflammaging (IL-8) and senescence (p16) in the in vitro model of skin photoaging using irradiated NHDFs. Inflammaging is associated with an upregulation of IL-8 in aged skin. ${ }^{9}$

Moreover, the redensifying effect of combined BK +VTE serum was demonstrated in an ex vivo human skin model of photoaging, with an increase in GAGs at the DEJ. In the skin, both GAGs and PGs have structural functions, acting as physiological regulators. ${ }^{15}$ In aging skin, GAGs are abnormally deposited on aberrant elastotic material which aggregates in the papillary dermis. ${ }^{17,18}$ This prevents a normal interaction with water and contributes to age-related skin dehydration. ${ }^{16}$ DEJs have several important functions including acting as mechanical support for the epidermis, providing adherence for the epidermal and dermal layers, and acting as a regulatory barrier for the passage of cells and macromolecules. ${ }^{29}$ Therefore, disruption of the DEJ following chronic UVA stress is likely to have functional consequences, and the 

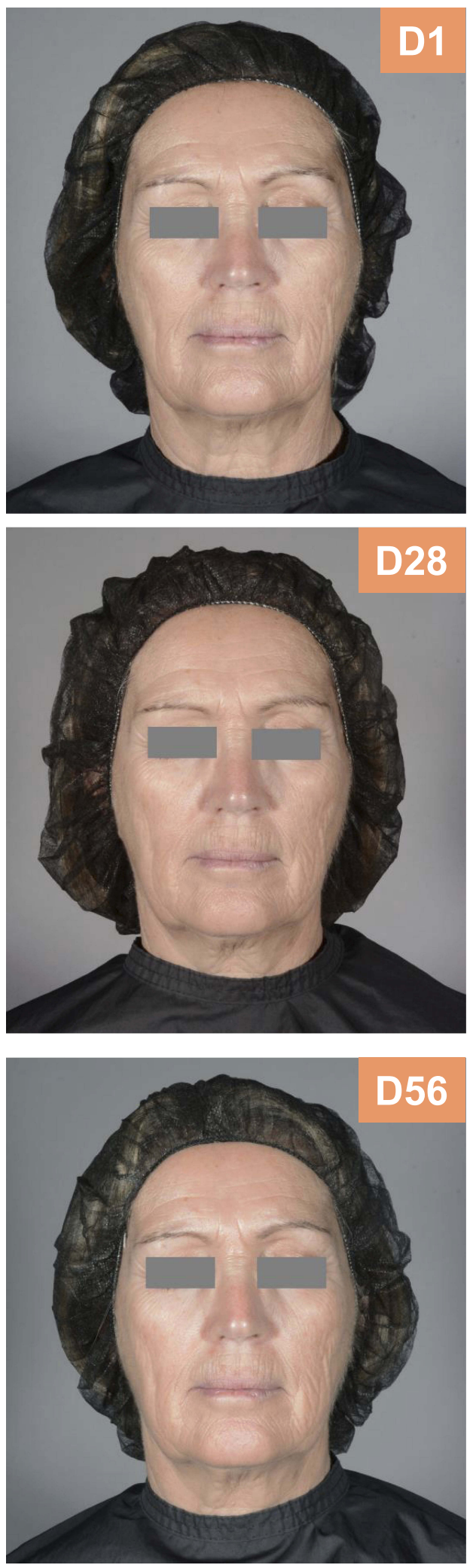

Figure 7 Full-face macrophotographs of radiance evaluation following application of the BK+VTE serum. Pictures show an improvement of radiance after 28 days $(+26 \%)$ and 56 days $(+44 \%)$ after twice daily use. redensifying effect of the BK+VTE serum is likely to ameliorate these abnormal effects at the clinical level.

In addition to the in vitro/ex vivo data presented in this study, the in vivo efficacy of the combination was demonstrated in female volunteers with naturally aged skin. The combination improved skin remodeling, firmness and radiance and may be used as part of an anti-aging strategy, particularly in women aged $\geq 45$ years with naturally aged skin. The serum was also well tolerated and safe, and data could be extended to other dermocosmetic products containing the BK+VTE combination. Indeed, although the current study reports data for the combination formulated as a serum (as the reference product), several other formulations of the combination (day cream, night balm, tinted day cream, eye contour cream) have also demonstrated in vivo anti-aging properties (data not shown).

In summary, the studies reported herein demonstrate the anti-aging properties of combined BK+VTE. Moreover, a serum combining both active ingredients has a good safety profile and shows clinical anti-aging efficacy, reducing ptosis, and increasing skin firmness and radiance. The combination of $\mathrm{BK}+\mathrm{VTE}$ could be used as part of a global anti-aging treatment strategy.

\section{Acknowledgments}

Medical writing/editorial assistance was provided, under the guidance of the authors, by David P. Figgitt PhD, ISMPP CMPPTM, Content Ed Net, with funding from Pierre Fabre Dermo-Cosmétique and Laboratoire Dermatologiques Avène, France.

\section{Funding}

The reported studies were funded by Pierre Fabre DermoCosmétique, France and Laboratoire Dermatologiques Avène.

\section{Disclosure}

All authors are employees of Pierre Fabre group, France. The authors report no other conflicts of interest associated with this work.

\section{References}

1. Farage MA, Miller KW, Elsner P, Maibach HI. Intrinsic and extrinsic factors in skin ageing: a review. Int J Cosmet Sci. 2008;30:87-95. doi:10.1111/j.1468-2494.2007.00415.x 
2. Makrantonaki E, Bekou V, Zouboulis CC. Genetics and skin aging. Dermatoendocrinol. 2012;4:280-284. doi:10.4161/derm.22372

3. Mukherjee S, Date A, Patravale V, Korting HC, Roeder A, Weindl G. Retinoids in the treatment of skin aging: an overview of clinical efficacy and safety. Clin Interv Aging. 2006;1:327-348. doi:10.2147/ ciia.2006.1.4.327

4. Uitto J. The role of elastin and collagen in cutaneous aging: intrinsic aging versus photoexposure. J Drugs Dermatol. 2008;7(2 Suppl): s12-s16.

5. Farage MA, Miller KW, Elsner P, Maibach HI. Structural characteristics of the aging skin: a review. Cutan Ocul Toxicol. 2007;26:343-357. doi:10.1080/15569520701622951

6. Zouboulis CC, Makrantonaki E. Clinical aspects and molecular diagnostics of skin aging. Clin Dermatol. 2011;29:3-14. doi:10.1016/j. clindermatol.2010.07.001

7. Cole MA, Quan T, Voorhees JJ, Fisher GJ. Extracellular matrix regulation of fibroblast function: redefining our perspective on skin aging. J Cell Commun Signal. 2018;12:35-43. doi:10.1007/s12079018-0459-1

8. Fisher GJ, Kang S, Varani J, et al. Mechanisms of photoaging and chronological skin aging. Arch Dermatol. 2002;138:1462-1470. doi:10.1001/archderm.138.11.1462

9. Strickland I, Rhodes LE, Flanagan BF, Friedmann PS. TNF-alpha and IL-8 are upregulated in the epidermis of normal human skin after UVB exposure: correlation with neutrophil accumulation and E-selectin expression. $J$ Invest Dermatol. 1997;108:763-768. doi:10.1111/1523-1747.ep12292156

10. Wolf J, Weinberger B, Arnold CR, Maier AB, Westendorp RG, Grubeck-Loebenstein B. The effect of chronological age on the inflammatory response of human fibroblasts. Exp Gerontol. 2012;47:749-753. doi:10.1016/j.exger.2012.07.001

11. Storey A, McArdle F, Friedmann PS, Jackson MJ, Rhodes LE. Eicosapentaenoic acid and docosahexaenoic acid reduce UVB- and TNF-alpha-induced IL-8 secretion in keratinocytes and UVB-induced IL-8 in fibroblasts. J Invest Dermatol. 2005;124:248-255. doi:10. 1111/j.0022-202X.2004.23543.x

12. Toutfaire M, Bauwens E, Debacq-Chainiaux F. The impact of cellular senescence in skin ageing: a notion of mosaic and therapeutic strategies. Biochem Pharmacol. 2017;142:1-12. doi:10.1016/j. bcp.2017.04.011

13. Jeyapalan JC, Ferreira M, Sedivy JM, Herbig U. Accumulation of senescent cells in mitotic tissue of aging primates. Mech Ageing Dev. 2007;128:36-44. doi:10.1016/j.mad.2006.11.008

14. Beauséjour CM, Krtolica A, Galimi F, et al. Reversal of human cellular senescence: roles of the p53 and p16 pathways. EMBO J. 2003;22:4212-4222. doi:10.1093/emboj/cdg417

15. Oh JH, Kim YK, Jung JY, Shin JE, Chung JH. Changes in glycosaminoglycans and related proteoglycans in intrinsically aged human skin in vivo. Exp Dermatol. 2011;20:454-456. doi:10.1111/j.16000625.2011.01258.x
16. Waller JM, Maibach HI. Age and skin structure and function, a quantitative approach (II): protein, glycosaminoglycan, water, and lipid content and structure. Skin Res Technol. 2006;12:145-154. doi:10.1111/j.0909-752X.2006.00146.x

17. Lee DH, Oh JH, Chung JH. Glycosaminoglycan and proteoglycan in skin aging. J Dermatol Sci. 2016;83(3):174-181. doi:10.1016/j. jdermsci.2016.05.016

18. Naylor EC, Watson RE, Sherratt MJ. Molecular aspects of skin ageing. Maturitas. 2011;69(3):249-256. doi:10.1016/j.maturitas.2011.04.011

19. Chaudhuri RK, Bojanowski K. Bakuchiol: a retinol-like functional compound revealed by gene expression profiling and clinically proven to have anti-aging effects. Int J Cosmet Sci. 2014;36:221-230. doi:10.1111/ics. 12117

20. Xin Z, Wu X, Ji T, et al. Bakuchiol: a newly discovered warrior against organ damage. Pharmacol Res. 2019;141:208-213. doi:10.1016/j.phrs.2019.01.001

21. Anuradha K, Shyamala BN, Naidu MM. Vanilla-its science of cultivation, curing, chemistry, and nutraceutical properties. Crit Rev Food Sci Nutr. 2013;53:1250-1276. doi:10.1080/10408398.2011.563879

22. Rouvrais C, Bacqueville D, Bogdanowicz P, et al. A new dermocosmetic containing retinaldehyde, delta-tocopherol glucoside and glycylglycine oleamide for managing naturally aged skin: results from in vitro to clinical studies. Clin Cosmet Investig Dermatol. 2017;10:35-42. doi:10.2147/CCID.S123575

23. Bacqueville D, Douki T, Duprat L, et al. A new hair follicle-derived human epidermal model for the evaluation of sunscreen genoprotection. $J$ Photochem Photobiol B. 2015;151:31-38. doi:10.1016/j.jphotobiol.2015.06.015

24. Dobos G, Lichterfeld A, Blume-Peytavi U, Kottner J. Evaluation of skin ageing: a systematic review of clinical scales. $\mathrm{Br} J$ Dermatol. 2015;172:1249-1261. doi:10.1111/bjd.13509

25. Karbacher S, Veit K. FaceSCAN ${ }^{3 \mathrm{D}}$ - photorealistic 3D images for medical applications. Presented at the 5th International Conference on 3D Body Scanning Technologies; 21-22 October; 2014; Lugano, Switzerland. Available from: https://www.3dbody.tech/cap/papers/ 2014/14010 52karbacher.pdf. Accessed 4 March 2019.

26. Kearney EM, Messaraa C, Grennan G, Koeller G, Mavon A, Merinville E. Evaluation of skin firmness by the DynaSKIN, a novel non-contact compression device, and its use in revealing the efficacy of a skincare regimen featuring a novel anti-ageing ingredient, acetyl aspartic acid. Skin Res Technol. 2017;23:155-168. doi:10.1111/srt.12314

27. Shetty P. Grey matter: ageing in developing countries. Lancet. 2012;379(9823):1285-1287. doi:10.1016/S0140-6736(12)60541-8

28. Darland AM, Chubb HA, Sachs DL, Helfrich YR. Patient interest in and familiarity with anti-aging therapies: a survey of the general dermatology clinic population. J Cosmet Dermatol. 2018;17:403-409. doi:10.1111/jocd. 12386

29. Briggaman RA, Wheeler CE Jr. The epidermal-dermal junction. J Invest Dermatol. 1975;65:71-84. doi:10.1111/1523-1747.ep12598050
Clinical, Cosmetic and Investigational Dermatology is an international, peer-reviewed, open access, online journal that focuses on the latest clinical and experimental research in all aspects of skin disease and cosmetic interventions. This journal is indexed on CAS.
The manuscript management system is completely online and includes a very quick and fair peer-review system, which is all easy to use. Visit http://www.dovepress.com/testimonials.php to read real quotes from published authors. 